\title{
Assessment of Protein-repellent and Antibacterial Capability of Dental Adhesive and Primer Containing 2-Methacryloyloxyethyl Phosphorylcholine in Primary Dentition
}

\author{
Shruthi B Patil ${ }^{1}$, Sayli R Khanvilkar ${ }^{2}$
}

\begin{abstract}
Aim: Resin composites are one of the favorite restorative materials used for treating early childhood caries (ECC). The biofilm accumulation at the microgaps between teeth and restoration with resin composite materials is more than other restorative materials. The main aim of this study was to evaluate the protein-repellent capability by measuring the total amount of protein adsorbed and anti-bacterial activity by measuring the production of lactic acid and colony-forming unit (CFU) counts of methacryloyloxyethyl phosphorylcholine (MPC) incorporated into dental adhesive and primer.

Materials and methods: The 2-MPC powder was added into dental primer and dental adhesive at mass fractions of $7.5 \%$ by mass individually. A dental plaque microcosm biofilm model with human saliva was used as the inoculum. Laury's method of protein estimation was used for measurement of total protein adsorbed. To evaluate the lactic acid production, enzymatic lactate dehydrogenase (LDH) method was employed. Tryptic soy blood agar and Mitis Salivarius agar were used for measuring the CFU counts for total microorganisms, total streptococci and only Streptococcus mutans.

Results: Results indicated that resin disks fabricated using dental adhesive and primer containing 7.5\% 2-MPC significantly reduced the protein adsorption, lactic acid production, and total streptococci and S. mutans counts $(p<0.05)$.

Clinical significance: Incorporation of protein repellent into dental adhesive and primer could help repel bacterial attachment and plaque buildup, assisting in the reduction of secondary caries.

Keywords: 2-Methacryloyloxyethyl phosphorylcholine, Dental adhesive and primer, Early childhood caries, Protein repellent, Streptococcus mutans. International Journal of Experimental Dental Science (2019): 10.5005/jp-journals-10029-1197
\end{abstract}

\section{INTRODUCTION}

The early childhood caries (ECC) has an impact on the child, starting from early infancy resulting in pain with impairment of form and function and also detrimental influence on the child's growth rate, body weight, and ability to thrive. As a pediatric dentist, the main aim is to eliminate the pain and infection, reduce the load of the bacterium Streptococcus mutans in the oral environment, and improve the well-being of the child. Also, it is important to recognize and treat the child for its appearance by restoring the carious teeth to an esthetically pleasing, functional, and natural looking relationship. Composite materials have been widely used to restore lesions due to their excellent esthetics and direct filling capabilities. However, studies have shown biofilm accumulation with composite materials. A number of times, micro gaps were observed at the interface of adhesive resin and primed dentin or between adhesive resin and the hybrid layer. ${ }^{1}$ Thus, a perfect seal and margin integrity with composite is compromised. These micro gaps harbor biofilms which is a source of infection, secondary caries, leading to restoration failure. ${ }^{2}$ Dental bonding agents facilitate the adherence of composite restoration to the tooth structure. It is of great benefit for the dental bonding agent to be antibacterial to oppose biofilms and secondary caries at the margins. Methacryloyloxyethyl phosphorylcholine (MPC) is a methacrylate with a phospholipid polar in the side chain and is one of the common biocompatible and hydrophilic biomedical polymers. Methacryloyloxyethyl phosphorylcholine shows
1,2Department of Pedodontics, SDM College of Dental Sciences and Hospital, Dharwad, Karnataka, India

Corresponding Author: Sayli R Khanvilkar, Department of Pedodontics, SDM College of Dental Sciences and Hospital, Dharwad, Karnataka, India, Phone: +91 8108420130, e-mail: saylirk1991@gmail.com

How to cite this article: Patil SB, Khanvilkar SR. Assessment of Proteinrepellent and Antibacterial Capability of Dental Adhesive and Primer Containing 2-Methacryloyloxyethyl Phosphorylcholine in Primary Dentition. Int J Experiment Dent Sci 2019;8(2):33-37.

Source of support: Nil

Conflict of interest: None

excellent resistance to protein adsorption and bacterial adhesion. ${ }^{2}$ Adding 2-MPC, a protein-repellent monomer in dental adhesive and primer with the property to repel and thus inhibit bacterial attachment, would be beneficial. Thus, the vital objective of this study was to evaluate 2-MPC incorporated in the dental adhesive and primer for its protein-repellent capability and antibacterial activity.

\section{Materials and Methods}

The research study was undertaken in the Department of Pedodontics in collaboration with Department of Microbiology. 
Institutional Review Board (IRB) of SDM Educational Society's Shri Dharmasthala Manjunatheshwara College of Dental Sciences and Hospital, Sattur, Dharwad, Karnataka, India, approved the study. Written informed consent approved by the Ethical Committee in accordance with the World Medical Association declaration of Helsinki was obtained from parents voluntarily participating for the study.

\section{Fabrication of Protein-repellent and Antibacterial Bonding Agent}

Scotchbond multipurpose primer and adhesive (SBMP; 3M, St. Paul, MN) was used as the parent system. The SBMP adhesive contained $60-70 \%$ of bisphenol-A glycol dimethacrylate (BisGMA) and $30-40 \%$ of 2-hydroxyethyl methacrylate (HEMA), tertiary amines and photoinitiator. The SBMP primer contained $35-45 \%$ of HEMA, $10-20 \%$ of a copolymer of acrylic and itaconic acids, and $40-50 \%$ water.

Methacryloyloxyethyl phosphorylcholine was synthesized via a method reported by Ishihara et al. and was commercially available (Sigma-Aldrich, St. Louis, MO) and was added at 7.5\% mass fraction into the Scotchbond multipurpose.

\section{Incorporation of 2-MPC into Bonding Agent}

The MPC powder at mass fractions of $7.5 \%$ was mixed into SBMP primer at a ratio of MPC/(SBMP primer + MPC). Primer was magnetically stirred with the help of magnetic stirrer at a speed of $150 \mathrm{rpm}$ for 2 hours by using a glass beaker and magnetic bead and was kept overnight to completely dissolve the MPC in the SBMP primer.

Similarly, 7.5\% MPC was mixed into SBMP adhesive study samples were divided into two main groups: group I-control group (SBMP primer and adhesive) and group II-test group (SBMP primer $+7.5 \%$ MPC and SBMP adhesive + 7.5\% MPC).

\section{Specimen (Resin Disks) Fabrication}

Study Sample

The study samples included 30 resin disks without 2-MPC in control group (group I) and 120 resin disks with 2-MPC into the test group. Each control group, i.e., group I1, group I2, and group I3 contained 10 resin risks. Each test group, i.e., group $\| 1$, group 112 , and group II 3 included 40 resin disks. Groups I1 and II1 were evaluated for protein adsorption, groups 12 and II2 for measurement of lactic acid production, and groups $\mathrm{I} 3$ and $I \mathrm{I} 3$ for measurement of colonyforming unit (CFU) counts.

\section{Size of the Resin Disks}

Each disk of $9 \mathrm{~mm}$ in diameter and $2 \mathrm{~mm}$ in thickness was fabricated.

\section{Steps for Fabrication of Resin Disks}

Cover plate of sterilized 96 well plates was used for fabrication of disk.

Primer of $10 \mu \mathrm{L}$ was applied to the bottom of each dent with the help of brush. After drying with a stream of air, $20 \mu \mathrm{L}$ of adhesive was applied similarly to the dent and photo polymerized for 30 seconds using a light curing unit (COLTOLUX LED). Then finally the mold was filled with composite (3M, ESPE) using Teflon-coated composite packing instrument and was light cured with the help of light curing unit (COLTOLUX LED) for 30 seconds.

After curing, the disks were immersed in $200 \mathrm{~mL}$ of distilled water, and with the help of magnetic stirrer the disks were magnetically stirred at a speed of $100 \mathrm{rpm}$ for 1 hour for removal of any uncured monomers.

Disks were sterilized with ethylene oxide and degassed for 3 days.

\section{For Collection of Saliva}

A dental plaque microcosm model was investigated for the viability of biofilm.

Saliva samples from 10 healthy children were collected. The donors were instructed to not to brush their teeth for 24 hours and were abstained from food and drink intake for 2 hours prior to donating saliva. Stimulated saliva was collected during paraffin chewing and was kept in ice at $-80^{\circ} \mathrm{C}$.

Inclusion criteria included children of age-group 3-6 years. Exclusion criteria are children with active caries or children having used antibiotics within the preceding 3 months or children suffering from any systemic diseases.

\section{Formation of Dental Plaque Microcosm Biofilm}

The saliva was added into the growth medium as inoculums. The growth medium, i.e., McBain medium contained mucin (type II, porcine, gastric) at a concentration of $2.5 \mathrm{~g} / \mathrm{L}$; bacteriological peptone, $2.0 \mathrm{~g} / \mathrm{L}$; tryptone, $2.0 \mathrm{~g} / \mathrm{L}$; yeast extract, $1.0 \mathrm{~g} / \mathrm{L}$; NaCl, $0.35 \mathrm{~g} / \mathrm{L} ; \mathrm{KCl}, 0.2 \mathrm{~g} / \mathrm{L} ; \mathrm{CaCl}_{2}, 0.2 \mathrm{~g} / \mathrm{L} ;$ cysteine hydrochloride, $0.1 \mathrm{~g} / \mathrm{L}$; hemin, $0.001 \mathrm{~g} / \mathrm{L}$; vitamin $\mathrm{K} 1,0.0002 \mathrm{~g} / \mathrm{L}$; at a $\mathrm{pH}$ 7. Inoculums of $1.5 \mathrm{~mL}$ were added to each well of 24 well plates containing a resin disk and was incubated at $37^{\circ} \mathrm{C}$ in $5 \% \mathrm{CO}_{2}$ for 8 hours. Then the disks were transferred to new 24 well plates filled with fresh medium and incubated. After 16 hours, the disks were transferred to new 24 well plates with fresh medium and incubated for 24 hours. This resulted in the formation of microcosm biofilms of 2-day-old.

\section{Measurement of Protein Adsorption by Laury's Protein Assay Method (Group I)}

Concentration of proteins was obtained by using Laury's method of protein estimation. For Laury's method, four reagents were used: Laury's solution A containing $2 \%(\mathrm{w} / \mathrm{v})$ sodium carbonate into $0.1 \mathrm{M}$ sodium hydroxide, Laury's solution B containing $0.5 \%$ copper sulfate $\left(\mathrm{CuSO}_{4} \cdot 6 \mathrm{H}_{2} \mathrm{O}\right)$ in $1 \%$ sodium potassium tartrate, Laury's solution $\mathrm{C}$ containing $50 \mathrm{~mL}$ of Laury's A mixed with $1 \mathrm{~mL}$ of Laury's B, and Laury's solution D containing $1 \mathrm{~N}$ Folin-Ciocalteu reagent

Each disk was first immersed in phosphate-buffered saline (PBS) for 2 hours and then in bovine serum albumin (BSA) solutions at $37^{\circ} \mathrm{C}$ for 2 hours in $2 \mathrm{~mL}$ sterile Eppendorf tubes. The concentration of BSA in the protein solutions was of $4.5 \mathrm{~g} / \mathrm{L}$. The disks were then rinsed with fresh PBS by stirring at a speed of 300 rpm for 5 minutes by using vortex mixer and later immersed in sodium dodecyl sulfate at $1 \mathrm{wt} \%$ in PBS and sonication at room temperature was done for 20 minutes to detach the BSA from the disk surfaces.

\section{Measurement of Lactic Acid Production Using LDH Enzymatic Method (Group II)}

Resin disks with 2-day biofilms were rinsed with cysteine peptone water (CPW) to remove any loose bacteria and then transferred to the 24 well plates having buffered peptone water (BPW) plus $0.2 \%$ sucrose and incubated at $37^{\circ} \mathrm{C}$ in the presence of $5 \% \mathrm{CO}_{2}$ for 3 hours for biofilms to allow lactic acid production. Lactate concentrations in the BPW solution were determined using lactate dehydrogenase (LDH) Erba enzymatic method. A microplate reader was used to measure the absorbance at $340 \mathrm{~nm}$ for the collected BPW. 


\section{Measurement of CFU Counts (Group III)}

Resin disks with 2-day biofilm were then transferred into sterilized Eppendorf tubes containing $2 \mathrm{~mL}$ CPW. By sonication and vortexing, biofilms were harvested from the resin disks. Three types of agar plates, i.e., tryptic soy blood agar for determining total microorganisms, Mitis Salivarius agar containing 15\% sucrose for determining total streptococci, and Mitis Salivarius agar plus $0.2 \%$ bacitracin per $\mathrm{mL}$ for determining $S$. mutans were used. Each agar plate is divided into five parts in which samples from different tubes were streaked. After serial dilution of the saliva samples, a loopful of sample $(10 \mu \mathrm{L})$ was spread over the surface of one section of an agar plate using an inoculating loop and incubation of agar plates was done at $37^{\circ} \mathrm{C}$ in $5 \% \mathrm{CO}_{2}$ for 24 hours. The number of colonies that grew was counted using the manual "click counter" method to calculate the CFU counts on each resin disk.

\section{Statistical Analysis}

The statistical analysis for the obtained data were completed. The statistical calculations were executed using the SPSS version 20.0 Statistical software. The following test was used:

- Independent $t$ test was used to compare the data between control and test group for all three parameters, i.e., measurement of total protein adsorbed, amount of lactic acid produced, and total CFU counts for total microorganisms, total streptococci and S. mutans.

- The level of significance $p$ value of less than 0.05 was considered significant.

\section{Results}

Table 1 shows the amount of protein adsorption on the surfaces of resin disks. Mean value of group I1 containing 2-MPC is showing lower value $(45.26 \pm 13.34)$ as compared to the group I1 (66.32 \pm 13.39) ( $p<0.001)$. Table 1 shows the amount of lactic acid produced by microorganisms present in the 2-day-old biofilms. The mean

Table 1: Comparison of protein adsorption onto resin disk surfaces between control group (group I1) and the test group (group II1) and lactic acid production of 2-day-old biofilm between control group (group I2) and the test group (group II2)

\begin{tabular}{|c|c|c|c|}
\hline Groups & Mean & $S D$ & pvalue \\
\hline \multicolumn{4}{|c|}{ Measurement of protein adsorbed onto resin disk surfaces } \\
\hline Group I1 & 66.32 & 13.99 & $0.0001^{*}$ \\
\hline Group II1 & 45.26 & 13.34 & \\
\hline \multicolumn{4}{|c|}{ Lactic acid production of 2-day-old biofilm } \\
\hline Group 12 & 16.89 & 3.90 & $0.0001^{*}$ \\
\hline Group II2 & -4.17 & 9.67 & \\
\hline
\end{tabular}

${ }^{*} p<0.05$ value of test group (group I12) containing 2-MPC is showing lower value $(-4.17 \pm 9.67)$ as compared to the control group (group I2) $(16.89 \pm 3.90)(p<0.001)$.

Table 2 shows the 2-day biofilm CFU counts for (A) total microorganisms, (B) total Streptococci, and (C) S. mutans. Adding 7.5\% MPC into dental adhesive and primer decreased the biofilm CFU counts in the test group (group II3) as compared to the control group (group I3).

Figure 1 shows the significant difference between the group 13 and group II3 for the CFU counts of total streptococci and S. mutans $(p<0.001)$.

However, difference between group I3 and group II3 for CFU counts of total microorganisms was not significant with $p$ value $>0.05$.

\section{Discussion}

Dental caries is one of the most common chronic oral diseases of childhood. The ECC acts as a source of pain and impairment and for some it results in serious infections, hospitalization, and even fatality. An essential prerequisite for the initiation and occurrence of caries is the formation of biofilms. ${ }^{3}$ Adsorbed proteins and protein coatings play a vital role in the attachment of oral bacteria to a material's surface. ${ }^{4-6}$ Considering biofilm formation and maturation, coaggregation and coadhesion play critical roles in bacterial colonization. Hence, for prevention of infectious diseases inhibition of adherence has been considered an efficient strategy. The management of dental caries is based upon the belief that surgical and restorative intervention is mandatory to discontinue the destruction of tooth structure due to caries. Materials such as glass ionomer cement, resin ionomer products, and improved resin based composite systems have been developed, which are having a profound impact on the restorations of primary teeth. Resin composites are very popular restorative materials as they offer the advantages of improved esthetics, more conservation of tooth structure, easy reparability, and direct filling capabilities. However, its disadvantages include incompatibility with moisture contamination during placement, potential marginal leakage, possible postoperative sensitivity, and a tendency to open or loose contacts causing accumulation of biofilms at the tooth restoration interfaces leading to the development of the secondary caries and failure of the restorations. Bernardo et al. conducted a 7-year clinical study comparing amalgam and resin-based composites and found composite to be an acceptable restorative material but the main cause of failure was the recurrent decay. ${ }^{7}$ Composite resins are bonded to the tooth by means of dental adhesive and primer through micromechanical bonding. A perfect seal at the restoration-tooth margin is often difficult to achieve due to the presence of microgaps between the tooth and the restoration. To improve, characterize, and understand enamel and dentin bonding, extensive studies have been performed. As bonding agents facilitate

Table 2: Comparison of colony-forming unit counts of 2-day biofilm for total microorganisms, total streptococci and Streptococcus mutans between control group (group I3) and the test group (group II3)

\begin{tabular}{|c|c|c|c|c|c|c|}
\hline \multirow[b]{2}{*}{ Groups } & \multicolumn{2}{|c|}{ Total microorganisms } & \multicolumn{2}{|c|}{ Total streptococci } & \multicolumn{2}{|c|}{ Streptococcus mutans } \\
\hline & Mean & $S D$ & Mean & $S D$ & Mean & $S D$ \\
\hline Group I3 & 216.60 & 69.89 & 151.00 & 47.16 & 25.20 & 14.96 \\
\hline Group II3 & 203.15 & 74.14 & 48.28 & 46.52 & 14.13 & 14.49 \\
\hline$p$ value & 0.6065 & & $0.0001^{*}$ & & $0.0001^{*}$ & \\
\hline
\end{tabular}

${ }^{*} p<0.05$ 


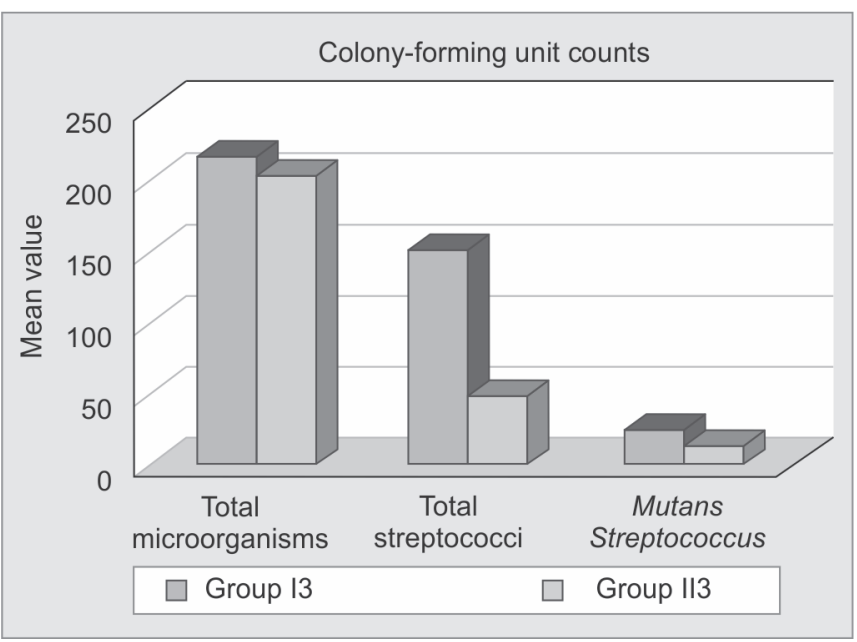

Fig. 1: Comparison of colony-forming unit counts of 2-day biofilm for total microorganisms, total streptococci and Streptococcus mutans between control group (group I3) and the test group (group II3)

the adhesion of composite restoration to the tooth structure, it will be favorable for the bonding agent to be antibacterial to prevent the formation of secondary caries at the tooth restoration interface. However, various previous studies have concluded that adsorption of salivary proteins from the physiological fluids significantly debilitate the antibacterial properties of the underlying surfaces. ${ }^{7}$ Protein adsorption onto the resin surfaces rendered the contactkilling mechanism less effective. Several studies have shown that a saliva-derived protein film on the cationic antibacterial surface reduced the original bactericidal effect. ${ }^{8-11}$ Hence, the main drawback of dental materials with contact-killing antibacterial activities is due to the reduction in the antibacterial effect by the adsorption of salivary proteins on the composite.

Thus, in our study, 2-MPC was the material of choice as it has protein-repellent activity and it could likely enhance the antibacterial effect of dental materials with contact-killing antibacterial activities.

Methacryloyloxyethyl phosphorylcholine is a methacrylate with a phospholipid polar in the side chain. And it is one of the common biocompatible and hydrophilic biomedical polymers. ${ }^{12,12,13}$ Methacryloyloxyethyl phosphorylcholine has shown exquisite resistance to protein adsorption and bacterial adhesion, and it was suggested that MPC is highly hydrophilic and there is an abundance of free water but no bound water in the hydrated MPC polymer. The presence of bound water causes protein adsorption. By contrast, the large amount of free water around the phosphorylcholine group is believed to detach proteins effectively, thereby repelling protein adsorption. ${ }^{14}$ The results obtained in our study showed a significant difference in the adsorption of proteins to the disks surfaces, formation of lactic acid, and CFU counts of streptococci and S. mutans between control group and test group (Table 1). In the control group, group 11, the salivary proteins present in the oral environment are adsorbed onto the resin composites providing a medium for attachment of bacteria and microorganisms. This attachment of proteins and microorganisms initiates the process of biofilm formation. Whereas in the test group, group II1, because of strong protein-repellent capability of 2-MPC, dental adhesive and primer containing 2-MPC has shown diminished bacterial adhesion to the resin surfaces. Various factors such as surface roughness and change in the chemical composition might affect the amount of protein adsorbed on to the resin disk surfaces. The Mylar strips were used to cover the resin surfaces in order to produce smooth and even surfaces for all the samples. Polishing and finishing of the resin disks was not done to maintain the standardization in the samples. Acidogenic bacterial growth typically occurs in plaque and biofilm. When biofilms forms at the tooth restoration margins, the demineralization of the enamel and dentin leads to secondary caries and restorative material fractures. ${ }^{15}$ Svanberg et al., through in vivo plaque studies, have shown that levels of lactic acid producing bacteria in the plaque present on restoration surfaces are significantly higher on resin composites than on the amalgam or glass ionomer cement $(\mathrm{GIC}) .{ }^{16}$ As the $S$. mutans count was low, the production of lactic acid was also reduced significantly in the group 12, a control group and group II2, a test group. As the protein-repellent capability of the 2-MPC reduced the accumulation of plaque, so the microorganisms were in direct contact with the restorative material.

In the present study, a dental plaque microcosm biofilm model was used which represents active attachment rather than a sedimentation biofilm model and uses saliva as inoculums.

Saliva was used as it is ideal for growing microcosm biofilms in vitro with the advantage of maintaining the complexity and heterogenicity of dental plaque in vivo. ${ }^{17}$ Together this provided new and relevant insights into the properties and dynamics of the dental plaque ecosystem. In our study, an artificial saliva-like culture medium (the McBain medium) was used for the biofilm culture experiments. $\mathrm{Li}$ et al. ${ }^{18}$ suggested that McBain artificial saliva medium produced medium-derived pellicles on the resin surfaces, which provided attenuating effects on biofilms similar to natural salivary pellicles. ${ }^{10}$ In the present study, Table 2 showed no significant difference with $p>0.05$ between the group I3, a control group, and group II3, a test group, for the count of total microorganisms present in the 2-day-old biofilm grown on the nonselective media, i.e., blood agar. This nonsignificant difference for the total microorganisms may be attributed to the use of nonselective simple media such as blood agar for culture that does not cause inhibition of any specific microorganisms. Also, the use of manual "click-counter" method for measurement of colonies may have missed few colonies due to overlapping. In our study, Mitis Salivarius agar culture plates containing 15\% sucrose were used to determine the total streptococci because Mitis Salivarius agar contains selective agents including crystal violet, potassium tellurite, and trypan blue which inhibit most gram-negative bacilli and most gram-positive bacteria except for streptococci, thus enabling the streptococci to grow. Cariogenic S. mutans is known to be resistant to bacitracin and this property was used to isolate $S$. mutans from the highly heterogeneous oral microflora for counting. Therefore, Mitis Salivarius agar culture plates plus 0.2 units of bacitracin per milliliter were used to determine S. mutans. ${ }^{19,20}$ The results obtained from this study were similar to the results obtained by Hirota and Zhang, that addition of 2-MPC into dental adhesive and primer, dental composite and resin modified GIC or coating it over biomedical device made up of polyethylene telephthalate causes less adsorption of proteins on to the surfaces leading to great reduction in the bacterial adhesion, CFU counts, and metabolic activity of dental plaque microcosm biofilms. ${ }^{6,21}$ The present study showed that it would be beneficial to add 2-MPC into dental adhesive and primer for providing strong protein-repellent and antibacterial activity in the dental adhesive and primer to combat the occurrence of secondary caries at the tooth restoration interface. 
Although it is important to inhibit bacterial growth and reduce occurrence of secondary caries to provide durable restoration, it is equally important for an adhesive and primer to be noncytotoxic to mammalian cell. Therefore, assessment of the cytotoxic behavior of MPC containing dental adhesive and primer must be performed in future studies.

\section{Conclusion}

Within the parameters of this study, it can be concluded that addition of 7.5\% 2-MPC resulted in the protein repellent and antibacterial activity against the occurrence of secondary caries at the interface between tooth and the restoration margin, and it could be highly beneficial in treating children with ECC and highrisk patients.

\section{Acknowledgment}

This study was supported by Department of Microbiology, Maratha Mandal's Nathajirao G Halgekar Institute of Dental Sciences and Research Centre, Belagavi, Karnataka, India.

\section{References}

1. Zhang N, Weir MD, Romberg E, et al. Development of novel dental adhesive with double benefits of protein-repellent and antibacterial capabilities. Dent Mater 2015;31(7):845-854. DOI: 10.1016/ j.dental.2015.04.013.

2. Zhang N, Melo MAS, Bai Y, et al. Novel protein-repellent dental adhesive containing 2-methacryloyloxylethyl phosphorylcholine. J Dent 2014;42(10):1284-1291. DOI: 10.1016/j.jdent.2014.07.016.

3. Daboor SM, Masood FSS, Al-Azab MS, et al. A review on Streptococus mutans with its diseases dental caries, dental plaque and endocarditis. Indian J Microbiol Res 2015;2(2):76-82.

4. Loesche WJ. Role of Streptococcus mutans in human dental decay. Microbiol Rev 1986;50(4):353-380. DOI: 10.1128/MMBR.50.4.353380.1986.

5. Leach SA, Hayes ML. A possible correlation between specific bacterial enzyme activities, dental plaque formation and cariogenicity. Caries Res 1968;2(1):38-46. DOI: 10.1159/000259542.

6. Gibbons RJ. Adherent interactions which may affect microbial ecology in the mouth. J Dent Res 1984;63(3):378-385. DOI: 10.1177/00220345840630030401.

7. Bernardo $M$, Luis $H$, Martin MD. Survival and reasons for failure of amalgam versus composite posterior restoration placed in a randomized control trial. J Am Dent Assoc 2007;138(6):775-783. DOI: 10.14219/jada.archive.2007.0265.

8. Muller R, Eidt A, Hiller KA. Influences of protein films on antibacterial or bacteriarepellen surface coatings in a model system using silicon wafers. Biomaterials 2009;30(28):4921-4929. DOI: 10.1016/ j.biomaterials.2009.05.079.

9. Leinfelder KF, Vaan WF. The use of composite resins on primary molars. Pediatric Dent 1982;4(1):27-31.

10. Li F, Weir MD, Fouad AF. Effect of salivary pellicle on antibacterial activity of novel antibacterial dental adhesives using a dental plaque microcosm biofilm model. Dent Mater 2014;30(2):182-191. DOI: 10.1016/j.dental.2013.11.004.

11. Awliya WY, El-Sahn AM. Leakage pathway of class V cavities restored with different flowable resin composite restorations. Oper Dent 2008;33(1):31-36. DOI: 10.2341/07-22.

12. Beyth N, Yudovin-Farber I, Bahir R. Antibacterial activity of dental composites containing quaternary ammonium polyethylenimine nanoparticles against Streptococcus mutans. Biomaterials 2006;27(21):3995-4002. DOI: 10.1016/j.biomaterials.2006.03.003.

13. Ishihara K, Iwasaki Y. Reduced protein adsorption on novel phospholipid polymers. J Biomater Appl 1998;13(2):111-127. DOI: 10.1177/088532829801300203.

14. Sibarani J, Takai M, Ishihara K. Surface modification on microfluidic devices with 2-methacryloyloxyethyl phosphorylcholine polymers for reducing unfavorable protein adsorption. Colloids Surf B Biointerfaces 2007;54(1):88-93. DOI: 10.1016/j.colsurfb.2006.09.024.

15. Dashper SG, Reynolds EC. Lactic acid excretion by Streptococcus mutans. Microbiology 1996;142(1):33-39. DOI: 10.1099/13500872142-1-33.

16. Svanberg $M, M j o r$ IA, Orstavik D. Mutans Streptococci in plaque from margins of amalgam, composite, and glass-ionomer restorations. J Dent Res 1990;69(3):861-864. DOI: 10.1177/00220345900690030601.

17. McBain AJ. Chapter 4: in vitro biofilm models: an overview. Adv App Microbiol 2009;69:99-132. DOI: 10.1016/S0065-2164(09)69004-3.

18. McBain AJ, Sissons C, Ledder RG. Development and characterization of a simple perfused oral microcosm. J Appl Microbiol 2005;98(3): 624-634. DOI: 10.1111/j.1365-2672.2004.02483.x.

19. Amerongen AV, Bolscher JG, Veerman EC. Salivary mucins: protective functions in relation to their diversity. Glycobiology 1995;5(8): 733-740. DOI: 10.1093/glycob/5.8.733.

20. Lewis AL. Phosphorylcholine-based polymers and their use in the prevention of biofouling. Colloids Surf B Biointerfaces 2000; 18(3-4):261-275. DOI: 10.1016/S0927-7765(99)00152-6.

21. Hirota K, Yumoto H, Miyamoto K, et al. MPC-polymer reduces adherence and biofilm formation by oral bacteria. J Dent Res 2011;90(7):900-905. DOI: 10.1177/0022034511402996. 